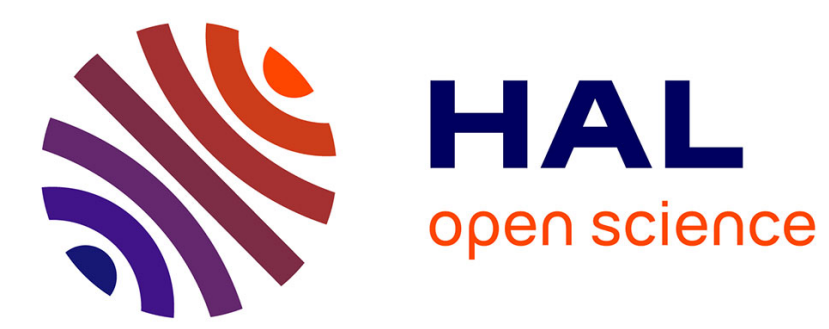

\title{
Beam waist measurement for terahertz time-domain spectroscopy experiments
}

\author{
Alexander Podzorov, Antoine Wojdyla, Guilhem Gallot
}

\section{To cite this version:}

Alexander Podzorov, Antoine Wojdyla, Guilhem Gallot. Beam waist measurement for terahertz timedomain spectroscopy experiments. Optics Letters, 2010, 35 (7), pp.901. 10.1364/OL.35.000901 . hal-00804604

\section{HAL Id: hal-00804604 \\ https://hal-polytechnique.archives-ouvertes.fr/hal-00804604}

Submitted on 1 Oct 2013

HAL is a multi-disciplinary open access archive for the deposit and dissemination of scientific research documents, whether they are published or not. The documents may come from teaching and research institutions in France or abroad, or from public or private research centers.
L'archive ouverte pluridisciplinaire HAL, est destinée au dépôt et à la diffusion de documents scientifiques de niveau recherche, publiés ou non, émanant des établissements d'enseignement et de recherche français ou étrangers, des laboratoires publics ou privés. 


\title{
Beam waist measurement for terahertz time-domain spectroscopy experiments
}

\author{
Alexander Podzorov, ${ }^{1,2}$ Antoine Wojdyla, ${ }^{1,2}$ and Guilhem Gallot ${ }^{1,2, *}$ \\ ${ }^{1}$ Laboratoire d'Optique et Biosciences, Ecole Polytechnique, CNRS, 91128 Palaiseau, France \\ ${ }^{2}$ INSERM U 696, 91128 Palaiseau, France \\ *Corresponding author: Guilhem.Gallot@polytechnique.edu
}

Received December 1, 2009; revised February 1, 2010; accepted February 4, 2010; posted February 24, 2010 (Doc. ID 120540); published March 17, 2010

\begin{abstract}
Classical masking aperture methods are found to be mostly inaccurate to determine the terahertz beam size in terahertz time-domain spectroscopy (TDS) experiments, owing to complex diffraction effects. Here, we present a simple and reliable method for measuring beam waists in terahertz TDS. It is based on the successive diffraction by an opaque disk followed by a small circular aperture. () 2010 Optical Society of America

OCIS codes: $140.3295,300.6495,050.1940$.
\end{abstract}

The terahertz range has become a popular domain in spectroscopy and imaging, mostly using single-cycle electromagnetic pulses and time-domain spectroscopy (TDS) [1-6]. As a result of the increasing importance of this technique, much interest has been applied to characterizing the terahertz beams: divergence, spatial or frequency modes, and polarization. Furthermore, the precise determination of the terahertz beam waist size and divergence is also fundamental for spectroscopy of inhomogeneous media (small samples [7], anisotropic samples, arrays of subwavelength apertures [8]) as well as for waveguide injection [9]. Cameras are still very challenging in the terahertz domain, and moving the detector in the plane of measurement is not easily achieved in TDS experiments [10]. In the visible or IR domains, circular apertures or moving slits are commonly used to determine the beam waists, using the overlap between the blocking screen and the laser beam [11]. However, in the terahertz domain, the role of diffraction has to be precisely studied, since the wavelength of the terahertz beams is much longer than in the visible, and diffraction could alter the measurements by the screens. Here, we show that diffraction plays a major role and that the classical circular aperture technique should not be used. Instead, we designed a simple and reliable method using circular apertures and disks, and we present experimental measurements.

We consider here a typical TDS system [2]. Both emitter and detector are considered to be fixed. Owing to the strong diffraction in the terahertz domain, only part of the emitted electromagnetic field is detected. This is in general not an issue for spectroscopy measurements, since the linear response of the TDS system can be subtracted from the sample measurements. But it is not true anymore when a diffracting object is put in the beam. First, the question of diffraction by a circular aperture can easily be addressed with the help of Fresnel's number $N$ $=4 a^{2} / \lambda d$, where $a$ is the radius of the aperture, $\lambda$ is the wavelength, and $d$ is the distance between the aperture and the plane of observation. The Fraunhofer (far-field) domain is achieved for $N \gg 1$, whereas the Fresnel (near-field) domain is valid for $N \ll 1$. Typical TDS systems encounter values of $N$ near unity [2]. As a consequence, (i) diffraction plays a major role, (ii) no domain approximations are allowed, and (iii) strong diffraction patterns are expected to take place after the apertures and through the detector. For symmetry reasons, we consider here circular diffracting apertures or disks. We assume a Gaussian beam profile as $E_{0}(r)=E_{0} \exp \left(-r^{2} / w^{2}\right)$, where $r$ is the axial polar coordinate and $w$ is the beam waist. Ideally, if the whole electromagnetic field transmitted through the aperture is collected by the detector (i.e., without diffraction losses), one would obtain a normalized integrated signal $S(a)=\left[1-\exp \left(-a^{2} / w^{2}\right)\right]^{2}$. The radius at half-maximum $R_{1 / 2}$ is then defined as $S\left(R^{(1 / 2)}\right)=1 / 2$ and would be proportional to the waist as

$$
R^{(1 / 2)}=\sqrt{\ln [\sqrt{2} /(\sqrt{2}-1)]} w \approx 1.108 w .
$$

The full calculations, taking diffraction losses into account, are conducted with Huygens' diffraction equation [12],

$$
\begin{aligned}
E(r)= & i \frac{2 \pi}{\lambda d} \exp \left(-i \pi \frac{r^{2}}{\lambda d}\right) \\
& \times \int_{0}^{a} \rho E_{0}(\rho) \exp \left(-i \pi \frac{\rho^{2}}{\lambda d}\right) J_{0}\left(2 \pi \frac{\rho r}{\lambda d}\right) \mathrm{d} \rho,
\end{aligned}
$$

where $E_{0}(\rho)$ is the electric field at the plane of the circular screen supposed infinitely thin, $E(r)$ is the electric field at the distance $d$ from the screen, and $J_{0}$ is the Bessel function of first kind. For complete simulation, the angular response of the detection, which merges the collection optics and the detector itself, is required. Since it is very difficult to precisely establish this angular response, it is fundamental that the experimental techniques are independent of the angular response of the detection. In our calculations, we assume a constant response of the detection up to the angle $\theta_{\max }$, and zero above, and we integrate the amplitude of the electric field. Then, the goal is to find experimental techniques that are independent of 
$\theta_{\max }$. The general measurement setup is found in the inset of Fig. 1. The radius of the first screen (aperture or disk) is $R_{1}$, and second aperture, of radius $R_{2}$, is introduced to take into account the optics (paraboloid mirror or lens) that partially collect the terahertz beam to the detector.

The calculations for the classical circular aperture masking technique are the black solid curves shown in Figs. 1 and 2. The aperture is positioned at equal distance between the two paraboloid mirrors. First, $R^{(1 / 2)}$ strongly depends on the wavelength (Fig. 1) owing to diffraction, in comparison to the expected value without diffraction [see Eq. (1)]. The observed modulations originate from the diffraction patterns at the second aperture and strongly affect the measurement. To deal with this problem, we reduced the size of the second aperture by adding a small circular aperture just in front of the focusing optics. In the ideal case of a very tiny aperture compared with the wavelength, one would observe a constant spherical diffraction pattern than is independent of $\theta_{\max }$. Calculations of $R^{(1 / 2)}$ versus $w$ are presented in Fig. 3 (black lines) and show unexpected saturationlike behavior. As a consequence, it is not possible to precisely determine the beam waist from $R^{(1 / 2)}$ measurements, since the uncertainty strongly increases at larger beam waists. Therefore, to thwart the diffraction, another strategy was considered. We replaced the first aperture by a solid disk. The new calculations are presented in Fig. 3 [gray (red online) lines]. The relationship between $R^{(1 / 2)}$ and $w$ is now almost linear and allows a precise determination of the beam waist. This strategy is also found to be almost independent of $\theta_{\max }$ as seen in Fig. 2 (dotted line) with a small second aperture, to be compared with a large second aperture (Fig. 2, dashed line).

Experimental measurements have been performed in two geometries. In the first one, the terahertz beam is focused in the middle of two paraboloid mir-

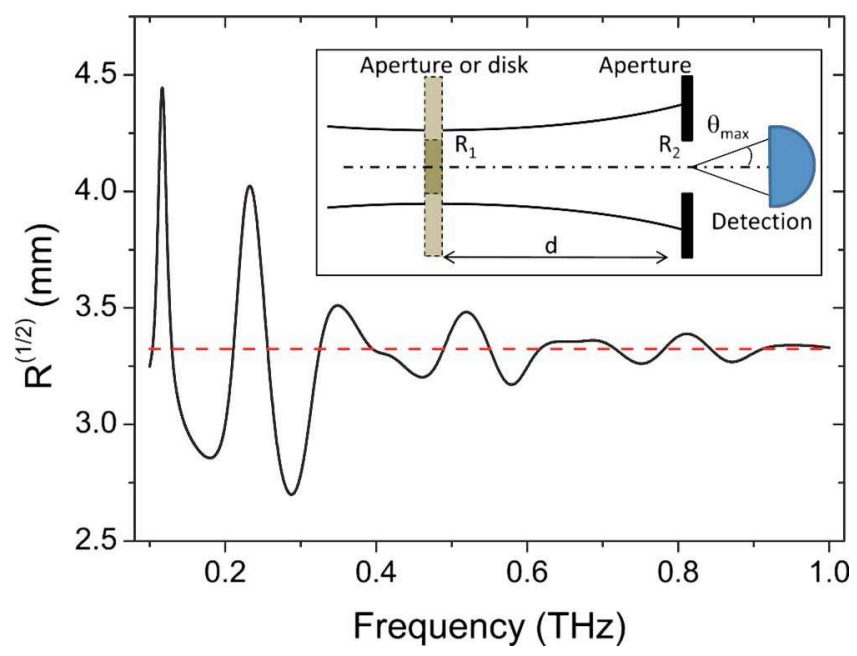

Fig. 1. (Color online) Half-maximum radius versus frequency for classical aperture/aperture strategy with a large second aperture: $R_{2}=32 \mathrm{~mm}, d=200 \mathrm{~mm}$, and $w=3 \mathrm{~mm}$. The horizontal line stands for theoretical value without diffraction: $R^{(1 / 2)} \approx 1.108 w$. Inset, experimental setup for both aperture/aperture and disk/aperture strategies.

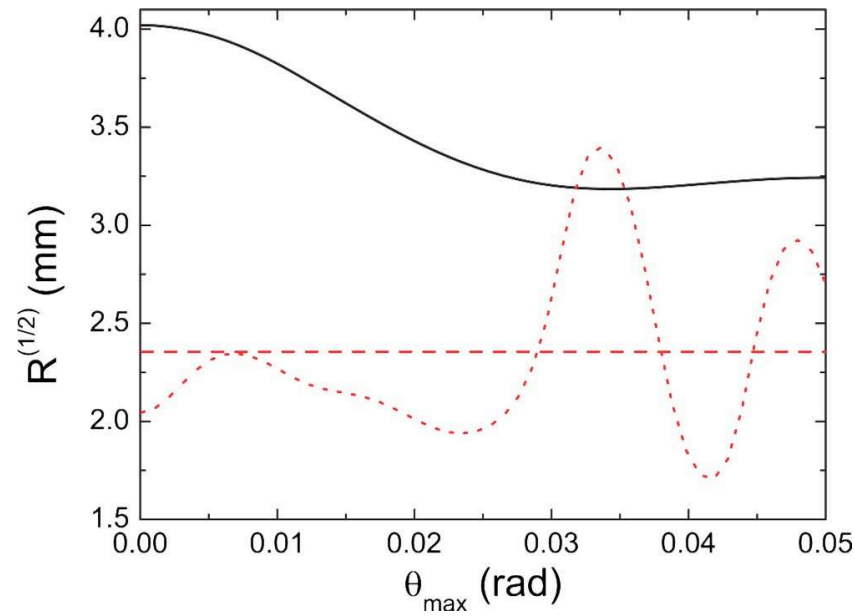

Fig. 2. (Color online) $R^{(1 / 2)}$ versus $\theta_{\max }$ for aperture/ aperture strategy (black solid line) and for disk/aperture strategy [gray (red online) lines] (dotted line: large aperture, $R_{2}=32 \mathrm{~mm}$; dashed line: small aperture, $R_{2}=2 \mathrm{~mm}$ ). In both cases, $d=200 \mathrm{~mm}, w_{0}=3 \mathrm{~mm}$, and $\nu=0.236 \mathrm{THz}$.

rors [13], in a $2 f-2 f$ geometry, where the distance between the emitter and the first paraboloid mirror is twice the focal length $f$ of the mirror $(2 f)$ as well as the distance between the second paraboloid mirror and the detector $(2 f)$, and the distance between the two paraboloid mirrors is $4 f$. In the second one, the terahertz beam is quasi-collimated between the mirrors in a $f-\infty$ geometry [2]. Several disks of various radius ranging from 1 to $15 \mathrm{~mm}$ have been used. The disks were punched out of aluminum foil and glued at the center of a $10-\mu \mathrm{m}$-thick cellophane film. This film was found to have no influence on the propagation of the terahertz radiation, and it was tightened on a rigid frame. A small circular aperture was positioned in front of the last paraboloid mirror. Transmission spectra for the different disks have been recorded by TDS [13]. The radius at half-maximum $R^{(1 / 2)}$ is then extracted from the measured spectra by

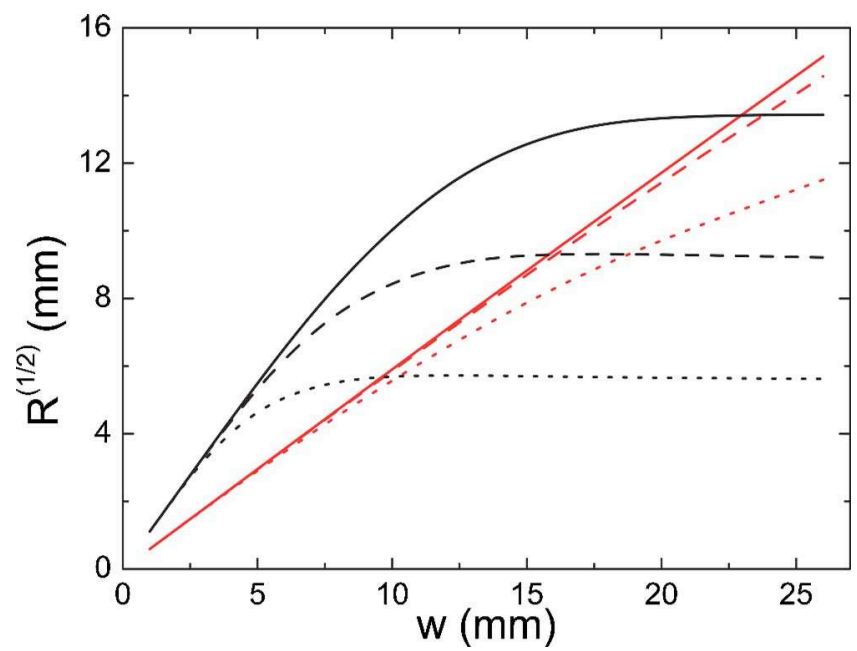

Fig. 3. (Color online) $R^{(1 / 2)}$ versus beam waist $w$, for circular aperture (black lines) and opaque disk [gray (red online) lines], at $1.1 \mathrm{THz}$ (solid lines), $0.45 \mathrm{THz}$ (dashed), and $0.2 \mathrm{THz}$ (dotted). In both cases, $d=500 \mathrm{~mm}, R_{2}=2 \mathrm{~mm}$, and $\theta_{\max }=0.001 \mathrm{rad}$. 
cubic interpolation. Results can be found in Fig. 4, for $2 f-2 f$ geometry [(a), solid line] and $f-\infty$ geometry [(b), solid line]. Using relationships similar to the ones calculated in Fig. 3 [gray (red online) red lines], we determined the corresponding beam waists versus frequency for the two geometries. Results are shown in Fig. 4 (dots). In the $2 f-2 f$ geometry [Fig. $4(\mathrm{a})$ ], one observes that the measured beam waist is almost independent of the frequency at the exception of an increase at smaller frequencies. This is expected from $2 f-2 f$ geometries, which are supposed to focus all the frequency components at the same point, except when diffraction is too strong [14]. It should be noted
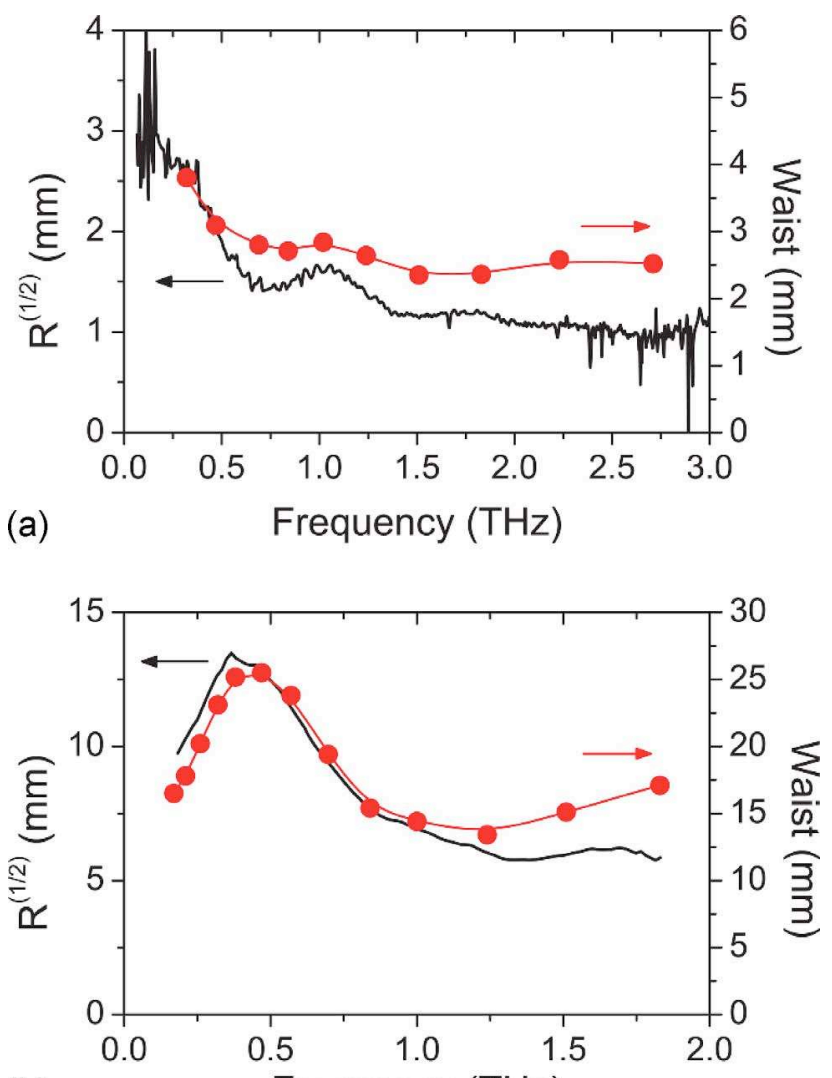

(b)

Frequency ( $\mathrm{THz})$

Fig. 4. (Color online) Experimental $R^{(1 / 2)}$ measurements (black solid lines) and calculated waist (dots) for $2 f-2 f$ (a) and $f-\infty$ (b) geometries. The focal length $f=120 \mathrm{~mm}$, and the distance between the two paraboloid mirrors in the $f-\infty$ geometry is $1000 \mathrm{~mm}$. that the measured $R^{(1 / 2)}$ shows different behavior with an apparent stronger frequency dependence, owing to diffraction artifacts during measurement. Experimental results for the $f-\infty$ geometry [Fig. 4(b)] show more complicated waist dependence with the frequency, probably owing to residual aberrations of the TDS setup.

As a conclusion, we demonstrated that diffraction plays a major role in the measurement of terahertz beam waist size using classical circular masking apertures in a TDS setup, and that the recovery of the beam waist may be very complicated. We present a simple and reliable technique using blocking disks and small apertures to overcome these difficulties. This technique is found independent of the angular response of the detection, and the relationships between the measured quantities and the beam waists allow a precise extraction of the data. Applied at several positions in the TDS setup, this technique could also provide the divergence of the terahertz beam.

\section{References}

1. D. Mittleman, Sensing with Terahertz Radiation, Optical Sciences (Springer, 2003).

2. D. Grischkowsky, S. R. Keiding, M. van Exter, and C. Fattinger, J. Opt. Soc. Am. B 7, 2006 (1990).

3. Q. Chen, M. Tani, Z. Jiang, and X. C. Zhang, J. Opt. Soc. Am. B 18, 823 (2001).

4. J. Shan, A. S. Welling, E. Knoesel, L. Bartels, M. Bonn, A. Nahata, G. A. Reider, and T. F. Heinz, Opt. Lett. 25, 426 (2000).

5. W. J. Padilla, A. J. Taylor, C. Highstrete, M. Lee, and R. D. Averitt, Phys. Rev. Lett. 96, 107401 (2006).

6. P. C. M. Planken, H. K. Nienhuys, H. J. Bakker, and T. Wenckebach, J. Opt. Soc. Am. B 18, 313 (2001).

7. N. C. J. van der Valk, W. A. M. van der Marel, and P. C. M. Planken, Opt. Lett. 30, 2802 (2005).

8. J.-B. Masson and G. Gallot, Phys. Rev. B 73, 121401(R) (2006).

9. G. Gallot, S. P. Jamison, R. W. McGowan, and D. Grischkowsky, J. Opt. Soc. Am. B 17, 851 (2000).

10. J. V. Rudd, J. L. Johnson, and D. M. Mittleman, Opt. Lett. 25, 1556 (2000).

11. J. A. Arnaud, W. M. Hubbard, G. D. Mandevil, B. D. de la Claviere, E. A. Franke, and J. M. Franke, Appl. Opt. 10, 2775 (1971).

12. A. E. Siegman, Lasers (University Science Books, 1986).

13. A. Podzorov and G. Gallot, Appl. Opt. 47, 3254 (2008).

14. J. C. G. Lesurf, Millimeter-wave Optics, Devices, and Systems (Adam Hilger, 1990). 